DOI: $10.21892 / 978-958-5547-34-6.3$

\title{
MEDICIÓN DE LA PERCEPCIÓN EMOCIONAL Y LA PERCEPCIÓN EMOCIONAL EN NIÑOS CON TDAH Y TOD
}

\author{
Rossy Jaraba ${ }^{1}$ \\ Ubaldo Ruiz ${ }^{2}$ \\ Jorge Navarro-Obeid ${ }^{3}$ \\ Corporación Universitaria del Caribe CECAR
}

\section{Resumen}

La presente investigación buscó determinar si existen diferencias significativas a nivel de las variables de cognición social, como la percepción de estados emocionales y del comportamiento social en niños con TDAH y TDAH/TOD, en comparación con sujetos normales, con edades comprendidas entre 11 y 15 años y entre los grados 6 a 9 de básica secundaria de una institución educativa regular de la ciudad de Sincelejo. La metodología se basó en un diseño prexperimental de tipo descriptivo comparativo con análisis estadístico del desempeño de los grupos de estudios de casos de niños con: TDAH (G2), TDAH/ TOD (G3) y niños controles (G1), con la aplicación de pruebas de cognición social: Test de falso paso y lectura mental de la mirada; igualmente, para la confirmación de diagnósticos se aplicaron Check List y la prueba de inteligencia Weschler (WISC-IV). Los resultados

1 Magister en Trastornos Cognoscitivos y del Aprendizaje (Uninorte), Especialista en Trastornos Cognoscitivos y del Aprendizaje (Uninorte). Docente y tutora universitaria en modalidad presencial, distancia y virtualidad en programas de psicología y licenciaturas. Correo: rossy.jaraba@cecar.edu.co Orcid: https://orcid.org/0000-0002-8527-9578.

2 Magister en gerencia del talento humano, master en salud mental y clínica social, especialista en psicología clínica, psicólogo. Docente de la corporación universitaria del caribe Cecar, facultad de humanidades y educación, programa de psicología. Correo: ubaldo. ruiz@cecar.edu.co Orcid: https://orcid.org/0000-0002-3918-1940.

3 Estudiante de primer año del Doctorado en Psicología de la Universidad Simón Bolívar de Barranquilla. Magister en Psicología. Corporación Universitaria del Caribe - CECAR, Sincelejo, Colombia. Correo: jorge.navarroo@cecar.edu.co Orcid: http://orcid.org/00000003-2160-5220. 
principales indican que existen diferencias en el procesamiento de la percepción emocional y del comportamiento social en los niños con TDAH y TDAH/TOD, en relación a niños sin estos trastornos; reflejando dificultades significativas en los niños con TDAH y TDAH/TOD en la percepción de emociones, discapacidad para procesamiento de contenidos afectivos, identificación y comprensión de las expresiones faciales de emociones. Asimismo, se encontró que los niños con TDAH y TDAH/TOD manifestaron dificultades en la detección de conductas inapropiadas de una situación social, incapacidad para interpretar las acciones, palabras, dobles sentidos e intenciones, dificultad para comprender los acontecimientos socialesde igual modo, incapacidad para predecir el impacto emocional de una acción en otra persona. Se evidenciaron carencias en el procesamiento e interpretación de la información social y la atención a las pistas sociales relevantes.

Palabras Clave: cognición social, TDAH, percepción emocional, percepción del comportamiento social.

\section{Abstract}

The present research sought to determine if there are significant differences at the level of social cognition variables such as the perception of emotional states and social behavior in children with ADHD and ADHD/TOD in comparison with normal subjects, with ages between 11 and 15 years and between grades 6 to 9 of basic secondary school of a regular educational institution in the city of Sincelejo. The methodology was based on a pre-experimental design of comparative descriptive type with statistical analysis of the performance of the groups of case studies of children with: ADHD (G2), ADHD/TOD (G3) and control children (G1), with the application of social cognition tests: test of false step and mental reading of the look; also, for the confirmation of diagnoses were applied Check List and the Weschler intelligence test (WISC-IV). The main results indicate that there are differences in the processing of emotional perception and social behavior in children with ADHD and ADHD/TOD in relation to children without these disorders, reflecting significant difficulties in children with ADHD and ADHD/TOD in the perception of emotions, disability to process affective content, identification and compression of facial expressions of emotions. It was also found that children with ADHD and ADHD/TOD, showed 
difficulties in detecting inappropriate behaviors of a social situation, inability to interpret actions, words, double meanings and intentions, difficulty in understanding social events and inability to predict the emotional impact of an action on another person. Shortcomings were evident in the processing and interpretation of social information and attention to relevant social clues.

Keywords: social cognition, TDAH, emotional perception, perception of social behavior.

\section{Introducción}

Una de las habilidades sociales que tenemos los seres humanos es realizar inferencias del comportamiento de los demás, esta capacidad es la que ha permitido al hombre desenvolverse socialmente en su ambiente, realizando ajustes y modificaciones en su conducta a partir del aprendizaje de sus experiencias previas, facilitando predicciones sobre el curso posible de una acción; esto es, la habilidad para percibir las intenciones y estados mentales de los otros (Brothers, citado en Rodríguez, Acosta y Rodríguez, 2011) se denomina cognición social.

La cognición social es un concepto que aún se encuentra en estudio conceptual y metodológico, sin embargo, en la literatura de la psicología se hace referencia al conjunto de operaciones mentales que subyacen a las interacciones sociales, que integran los procesos implicados en la percepción, interpretación y generación de respuestas ante las intenciones; disposiciones, así como conductas de los demás (Ostrom; Brothers, como se cita en Ashford \& LeCroy, 2009). Es decir, se refiere al conjunto de proceso cognitivos que a partir de su funcionamiento dan cuenta de cómo la gente piensa sobre sí misma y los demás, asimismo sobre las situaciones e interacciones sociales (Penn, Corrigan, Bental, Racenstein, y Newman, 1997).

Partiendo de esta idea es posible atribuir estados mentales tanto a sí mismo como a los demás, es decir: ser capaz de entender el rol del otro y en cierta forma ponerse en el lugar de esa persona; de esta forma, se postula la "teoría de la mente", la cual es una variable de la cognición social que hace referencia a las inferencias que un individuo puede hacer sobre las actitudes, pretensiones o sentimientos de los demás, estas constituyen una 
teoría, pues aun cuando no son estrictamente comprobables, si permiten realizar predicciones sobre el comportamiento del otro, y de esta manera actuar de la manera más conveniente en una determinada situación social (Perner \& Lang, 1999; Baron-Cohen, \& Wheelwright, 2004; Pineda y Puentes, 2013).

Las variables a estudiar desde la cognición social —objeto de estudio de la presente investigación- son la percepción emocional y la percepción del comportamiento social; la primera apunta a la capacidad de identificar y hacer una lectura de nuestros propios sentimientos y emociones, de igual modo a la de los demás, así como a la capacidad de ser flexible y adaptarse a múltiples facetas afectivas. Esto requiere reconocer las emociones expresadas, tanto a nivel verbal como gestual, en el rostro y en cuerpo de los demás.

Consecuentemente, se puede distinguir y asignar un valor o contenido emocional a los acontecimiento o situaciones sociales, para así describir, expresar y designar una etiqueta a lo que se está percibiendo (Extremera y Fernández, 2003). La segunda variable en estudio, percepción del comportamiento social, se refiere a elementos asociados a valorar reglas y roles sociales, así como para evaluar el contexto social. Estos procesamientos sociales se encuentran fundamentados en procesos perceptivos básicos que permiten la orientación de la atención hacia señales o claves sociales por ende, ayudan a la interpretación de situaciones en las que la persona interactúa. La percepción del comportamiento social, implica dos fases, una fase de identificación o categorización de la conducta, y otra, que permite decidir si la conducta observada es debido a estados emocionales estables o a elementos situacionales (Bellack, Blanchard \& Mueser; Newman y Uleman, citados en Ruiz-Ruiz, García \& Fuentes, 2006).

En síntesis, la cognición social tiene un papel importante en el desarrollo de relaciones generadoras de confianza y empatía en los grupos sociales. Lo anterior nos lleva a señalar que la cognición social es una de las claves fundamentales para que en la sociedad se evidencie el establecimiento de convivencias sanas y pacíficas que contribuyan a la vinculación afectiva. Cuando tenemos una comprensión de los sentimientos del otro, nos permite desarrollar conductas pro sociales y altruistas (Montoya \& Arango, 2015).

Butman, Abel y Allegri (2003), mencionan la existencia de estructuras anatómicas que se relacionan con dicha capacidad, dentro de 
las cuales se encuentran: las cortezas sensoriales superiores encargadas de las representaciones perceptuales de los estímulos; así como el contenido emocional que procesa la amígdala; las regiones corticales como el frontal izquierdo, parietal derecho y región del cíngulo que se relacionan con la forma de actuar en un contexto social y la corteza orbitofrontal encargada de la toma de decisiones y el razonamiento social; siendo así, es claro que el funcionamiento ejecutivo resultante de la actividad de áreas prefrontales se encuentra estrechamente ligado con la capacidad para interactuar adecuadamente con el otro, ello implica a su vez el potencial para coordinar los procesos mentales, dirigir la motivación, controlar los impulsos y monitorear la conducta en pro de una mejor adaptación a las demandas del entorno.

Hay numerosos estudios que recogen la presencia de alteraciones en la cognición social en diversos trastornos y/o déficits. Cuando existe déficits o ausencia de cognición social se ven mermadas varias competencias sociales y personales en el individuo. Es el caso de los sujetos que presentan trastornos de conducta, tal es el caso del Trastorno por déficit de atención e hiperactividad (TDAH) y el Trastorno oposicionista desafiante (TOD), en los cuales se encuentran ausencias de habilidades sociales como la empatía y la regulación y/o control inhibitorio (Restrepo, Arana, Alvis \& Hoyos, 2015).

Ambos trastornos se caracterizan por una alteración de origen poligenético que tienen una base neurológica, caracterizada principalmente por la disfunción de los sistemas fronto-estriados relacionada con el control de impulsos y la autorregulación emocional, esto se refleja en una clara dificultad para relacionarse adecuadamente con las demás personas (Ramírez, 2015).

Se han realizado varias investigaciones y estudios donde se da cuenta de la ausencia de la cognición social en personas con TDAH (García, 1999; Clark, Prior \& Kinsella, 2002; Rapport, Friedman, Tzelepis y Van Voorhis, 2002; Yuil y Lion, 2007), todos estos apuntan a que las habilidades sociales se encuentran claramente deterioradas, ya sea por dificultades atencionales, por un sesgo en la percepción del comportamiento social o por el pobre control de impulsos y regulación emocional. Otras investigaciones han hecho referencia a que los niños con TDAH presentan un déficit en el proceso comunicativo no verbal, específicamente, en el proceso perceptivo, 
en este sentido, el reconocimiento de expresiones faciales de emociones se vería afectado, por lo que una incorrecta identificación de estas resulta fundamental para establecer y regular relaciones interpersonales (Pardo, Fernández-Jaén \& Fernández-Mayoralas, 2009).

Todos estos estudios apuntan a que las habilidades sociales en estos trastornos se encuentran claramente deterioradas, ya sea por dificultades atencionales, por un sesgo en la percepción del comportamiento social o por el pobre control de impulsos y regulación emocional.

En estudios realizados se encontró que entre los aspectos más afectados en la cognición social de niños con TDAH se encontraban "la dificultad para responder a las demandas planteadas en la interacción, la baja tolerancia a la frustración y la conducta agresiva, que lleva a los sujetos que padecen este tipo de trastorno al rechazo y al aislamiento social" (Castaño, 1999, p. 21). Se expone que en los niños con TDAH existe un déficit en habilidades de mediación verbal o autoinstrucciones, relacionado con la dificultad para aprender las habilidades sociales adecuadas que involucren respuestas (Mellado, Martínez y Tello, 2013; Orjales, 2007).

De igual forma, estudios realizados apuntan a que entre el 50\% $70 \%$ de niños con TDAH presentan dificultades en la relación social con su par, y los problemas sociales aumentan el riesgo de padecer alteraciones emocionales, así como conductuales, a lo largo del ciclo vital (RosellóMiranda, Berenguer-Forner, Baixauli-Fortea \& Miranda-Casas, 2016).

Por su parte, la cognición social en los niños con TOD, ha sido poco estudiada, sin embargo, se considera que estos individuos, en inicio, empiezan a tener comportamientos coactivos en otras interacciones sociales, conduciéndolos a conductas agresivas y destructivas con sus compañeros y adultos. Posterior a los intercambios coactivos recurrentes, muestran formas problemáticas de procesar la información social; esto se soporta en las deficiencias encontradas en la comprensión lingüística, la memoria verbal y la fluidez verbal (Fonseca-Parra \& Rey-Anacona, 2013), lo que indica las dificultades en el procesamiento del contenido verbal, primordial para la regulación emocional, cognoscitiva y conductual entre los niños con problemas de comportamiento (Eme, 2007; Rubia, 2011).

Desde esta misma perspectiva, es claro que la capacidad de entender y comprender las intenciones, ideas, sentimientos de las otras personas, se ve 
afectada en niños con trastornos de conducta (TDAH y TOD, en particular) presentando alteraciones representadas en las actitudes, hostiles, agresivas, desobedientes, oposicionista, desorganizadas hacia los demás personas, de este modo, es probablemente que la impulsividad sea un factor vinculante, para la coexistencia, de los dos trastornos (TDAH y negativismo desafiante).

Igualmente, estudios recientes muestran resultados donde se puntualiza que dificultades encontradas en las funciones ejecutivas en niños con TND/TOD (Trastorno Negativista Desafiante) generan alteraciones en la cognición social, lo que da cuenta de déficits en las capacidades de regulación emocional y el uso de estrategias óptimas para la planeación y ejecución de comportamientos adecuados, lo que lleva a que tengan conductas agresivas y problemas en las repuestas prosociales con los otros (Ison-Zintilini \& Morelato, así como es citado por Restrepo et al., 2015).

En la presente investigación se tuvo como objetivo principal determinar si existen diferencias significativas a nivel de las variables de cognición social como la percepción de estados emocionales y del comportamiento social en niños con TDAH y TDAH/TOD en comparación con sujetos normales, debido a la necesidad de comprender cómo la alteración de dichas capacidades puede interferir en el adecuado desarrollo de las relaciones interpersonales; "numerosos estudios han documentado que los niños con TDAH experimentan dificultades interpersonales y baja competencia social" (Pardos, Fernández-Jaén, Fernández-Mayoralas, 2009, p. 107); generado por su incapacidad para detectar en el otro pistas sociales que le permitan predecir estados emocionales y actuar acorde a la situación. Igualmente, se ha encontrado que en las conductas oposicionistas asociadas al TDAH (TDAH/TOD) existe un mayor riesgo de desarrollar déficit significativos con relación a la competencia social, sobretodo, en la cooperación con pares y adultos, pues implica tanto el seguimiento de reglas, como la necesidad de generar respuestas adecuadas a las expectativas sociales del entorno.

Siendo así, se considera de gran importancia estudiar tanto en niños sanos como en niños con TDAH y TDAH/TOD, la manera como su cerebro responde a las diversas demandas sociales, a partir de la forma en que perciben las expresiones emocionales y comportamientos sociales en su relación con el otro (Pardos et al., 2009). A partir de lo anterior, se pretender dar respuesta a la siguiente pregunta de investigación: ¿Existen diferencias 
en la cognición social en niños con TDAH y TDAH/TOD en comparación con niños sin patología?

\section{Metodología}

En este apartado se expone la perspectiva metodológica, se especifica el tipo de diseño, la población y muestra; instrumentos y procedimientos, entre otros aspectos que orientaron la realización del estudio.

\section{Diseño}

Esta investigación se basa en un paradigma positivista de tipo cuantitativo, con un diseño descriptivo transversal con análisis de casos y controles (Hernández, Fernández y Baptista, 2014). El alcance del estudio fue de tipo descriptivo comparativo con análisis de grupo control y grupo experimental, con la finalidad de identificar diferencias en la variable de cognición social en los grupos de niños con TDAH y TDAH/TOD y la ausencia de las mismas (Hurtado de Barrera, 2012).

\section{Población y muestra}

La población de esta investigación corresponde a estudiantes de $6^{\circ}$ a $9^{\circ}$ grado, con edades comprendidas entre 11 y 15 años, que asisten a aulas regulares de clases en la ciudad de Sincelejo. La selección de la muestra fue intencional, dado que se tuvo en cuenta la caracterización de los sujetos a estudiar. El tamaño de la muestra obtenido fue de 30 estudiantes de una institución educativa de estrato socio-económico medio (3 y 4), con un promedio de edad de 12,5. En la tipificación de la muestra los sujetos fueron distribuidos en 3 grupos de estudio, los cuales se encontraban equiparados por edad, grado escolar y coeficiente intelectual, como se indica en la Tabla 1 .

Cada uno de los grupos estaba conformado por 10 sujetos:

- Grupo 1: sujetos controles sin ningún tipo de patología.

- Grupo 2: sujetos con TDAH tipo Combinado.

- Grupo 3: sujetos con TDAH-C/TOD. 
Tabla 1

Datos clínicos y Sociodemográficos

\begin{tabular}{lccc}
\hline & Edad & CI & $\begin{array}{c}\text { Grado } \\
\text { Escolaridad }\end{array}$ \\
\hline Control & 12,5 & 95 & 7,7 \\
Tdah & 12,5 & 94,3 & 7,3 \\
tod & 12,6 & 94,2 & 7,5 \\
\hline
\end{tabular}

Fuente: elaboración propia (2018).

\section{Instrumentos}

Los instrumentos utilizados fueron un Check list elaborado con los criterios diagnósticos del DSM-IV para identificar sintomatología presente en el trastorno por déficit de atención e hiperactividad y el Trastorno oposicionista desafiante.

Escala de inteligencia Weschler (WISC-III), para evaluar las capacidades cognitivas. Cuenta con 13 subtests organizados en dos escalas: verbal y de ejecución, que permiten calcular un CI total; además de los índices de Comprensión Verbal, Organización Perceptual, Ausencia de Distractibilidad y Velocidad de Procesamiento.

Test de falso paso (Faux pas): (Stone, Baron-Cohen, Knoght, 1998). Mide la Capacidad de percepción del comportamiento social a partir de la detección de intenciones y conductas incorrectas. El test consiste en 10 historias que contienen un FP (Falso Paso/error) social y 10 historias control que contienen un conflicto menor que no constituye un FP, y se encuentran agrupadas en orden aleatorio.

Los participantes respondieron preguntas que implicaran detección y comprensión del FP; asimismo dos preguntas de control que evalúan la comprensión en general. Con estas preguntas se mide la habilidad de conocer que quien cometió el FP, no sabía al momento de cometerlo que no debía haberlo dicho y que no tuvo la intención de incomodar a su oyente (Butman, et al, 2007).

Lectura mental de la mirada: (Baron-Cohen, Wheelwright y Hill, 2001) miden la habilidad de reconocer el estado mental de una persona a través de la expresión de su mirada, aparece alrededor de la adolescencia y hace énfasis en el aspecto visuoperceptivo, minimizando la demanda 
de memoria, de FE y contextual. Esta tarea emplea 36 fotografías de rostros de ambos sexos, en blanco y negro, de la misma región ocular. El sujeto debe "leer la mirada" y elegir una de cuatro palabras impresas que representan términos referidos al estado mental, que a su juicio exprese mejor lo que siente la persona fotografiada. Los términos empleados para describir el estado mental eran previamente explicados por el examinador y se encontraban definidos en un glosario disponible si el examinado lo requería.

\section{Procedimiento}

Para el desarrollo de la presente investigación se seleccionó la muestra de una población de estudiantes de 11 a 15 años pertenecientes a los grados de $6^{\circ}$ a $9^{\circ}$. Para lo cual se contó con la colaboración de los planteles educativos Institución Educativa Técnico Industrial Antonio Prieto de la ciudad de Sincelejo y el Instituto Pestalozzi de la ciudad de Barranquilla, resulta oportuno acotar que se obtuvo el respectivo consentimiento informado. Como primer paso, se solicitó la colaboración de los profesores de aula para detección de individuos con alto riesgo de presentar los trastornos de TDAH y TOD, a partir de una prueba de tamizaje (check list) conformado por los criterios diagnósticos para dichas patologías según el DSM IV TR. Posteriormente, se procede a la confirmación del diagnóstico por parte del especialista.

Una vez confirmado el diagnóstico se seleccionaron los sujetos que cumplieran con los criterios de inclusión de la investigación: tipo de predominio, sexo, edad y CI promedio (85-130), por lo que, seguidamente, se aplicó la escala de inteligencia de Weschler (WISC III), a cada uno de los niños que cumplieran los 3 primeros criterios y así descartar bajo coeficiente intelectual.

Con la muestra seleccionada se aplicaron las pruebas de cognición social: Faux Pas y Lectura de miradas. En la recolección de los datos con la primera prueba se valoró la percepción de las expresiones emocionales y con la segunda se hizo una valoración de la capacidad perceptiva del comportamiento social de los sujetos pertenecientes a cada uno de los grupos de estudio. Con dichos resultados se contrastan los hallazgos de la investigación, con estudios previos y se analizan a la luz de la teoría. Por último, se extrajeron las conclusiones que se obtuvieron en la investigación 
y se expusieron las recomendaciones pertinentes en cuanto a futuras investigaciones en el área.

\section{Análisis de la información}

Se realizó un análisis estadístico por medio del estadígrafo U de Mann Whitney, para determinar la existencia de diferencias entre las variables clínicas y demográficas de la muestra; no se encontró diferencia en edad, el nivel de escolar y el índice de inteligencia actual. Finalmente, se escogieron de forma intencional 30 estudiantes para la muestra, divididos en grupos de 10 participantes, cada uno según la caracterización de la misma: Grupo Control sin patología (G1), Grupo con TDAH (G2) y Grupo con TDAH/ TOD (G3).

Para analizar el desempeño obtenido en dichas pruebas, se llevó a cabo un análisis descriptivo de las medias de las puntuaciones directas obtenidas, igualmente, se utilizó el estadígrafo U de Mann Whitney para establecer las diferencias entre las puntuaciones de los diferentes grupos de análisis, y determinar si esta se consideraba significativa. El nivel de significancia que se utilizó para la comparación de las medias de puntuación de los grupos de estudio fue de $\mathrm{P}<0,05$.

\section{Resultados}

En este apartado, se presentan los resultados del estudio, estos se engloban en dos aspectos: Rendimiento General en Pruebas de Cognición Social y la Comparación de Grupos en las variables de Percepción Emocional y Percepción Social (Cognición Social).

\section{Rendimiento general en pruebas de cognición social}

A continuación, se describen los resultados relacionados con las variables de estudio: percepción de expresiones emocionales y percepción del comportamiento social en los diferentes grupos de estudio (Gl: Control, G2: TDAH, G3: TDAH/TOD). 


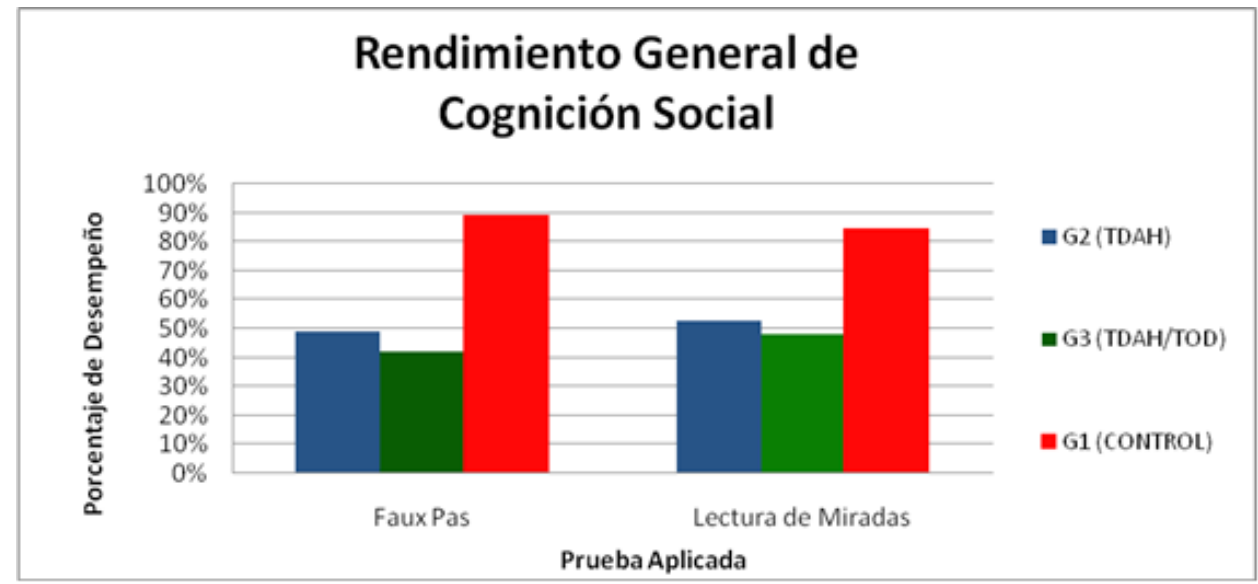

Figura 1. Porcentaje de Rendimiento de los grupos 1, 2, 3 en las pruebas de cognición social.

En la Figura 1 se observa que el Gl (control) obtuvo el 90\% en el nivel desempeño en la ejecución de las pruebas Faux Paux y Lectura de miradas, lo que indica que esta población tiene un adecuado procesamiento en la percepción de las expresiones emocionales y del comportamiento social; mientras que el G2 obtiene un $48 \%$ en los resultados del test de Faux Paux y 53\% en el test de lectura de miradas, lo que permite dar cuenta de un desempeño promedio en este grupo. Mientras que en el G3 la ejecución tuvo una notoria disminución en el rendimiento en ambas pruebas, proyectando $40 \%$ en la primera y $45 \%$ en la segunda prueba. Se evidenció disminución en el rendimiento ante las pruebas en los grupos 2 y 3 en comparación con los grupos 1 .

\section{Comparación de grupos en las variables de percepción emocional y percepción social (cognición social)}

Al realizar un análisis comparativo de los grupos mediante estadígrafo U Man-Whitney, se encuentran diferencias significativas entre G1>G2>G3; siendo solo significativa las diferencias entre G1-G2 y Gl-G3, como se muestra en la Tabla 2; indicando que existen diferencias en el procesamiento de la percepción emocional y del comportamiento social en los niños con TDAH y TDAH/TOD, en relación a niños sin estos trastornos; esto muestra las alteraciones del grupo G2 y G3 al realizar tareas de identificación de conductas sociales y al realizar tareas de reconocimiento de emociones a partir de un indicador facial. 
Tabla 2

Diferencias establecidas en la comparación de los grupos de estudio a partir del estadigrafo U de Mann Whitney

\begin{tabular}{lcccccc}
\hline Prueba & \multicolumn{2}{c}{$\begin{array}{c}\text { Comparación } \\
\text { Grupos 1 y 2 }\end{array}$} & \multicolumn{2}{c}{$\begin{array}{c}\text { Comparación } \\
\text { Grupos 1 y 3 }\end{array}$} & \multicolumn{3}{c}{$\begin{array}{c}\text { Comparación } \\
\text { Grupos 2 y 3 }\end{array}$} \\
\hline & $\begin{array}{c}\text { U Man- } \\
\text { Whitney }\end{array}$ & P & $\begin{array}{c}\text { U Man- } \\
\text { Whitney }\end{array}$ & P & $\begin{array}{c}\text { U Man- } \\
\text { Whitney }\end{array}$ & P \\
\cline { 2 - 8 } & 2,500 & 0,000 & 0,000 & 0,000 & 43,000 & 0,596 \\
Faux Pas \\
$\begin{array}{l}\text { Lectura de } \\
\text { Miradas }\end{array}$ & 6,500 & 0,001 & 5,000 & 0,001 & 41,000 & 0,494 \\
\hline
\end{tabular}

Fuente: elaboración propia (2018).

\section{Discusión}

Al medir la cognición social en los grupos de estudio (TDAH, TDAH/ TOD, y grupo control) se encontró que los rendimientos de ejecución en ambas variables de investigación (percepción de expresiones emocionales y percepción del comportamiento social) de Grupo 2 (G2) y Grupo 3 (G3) fueron significativamente inferiores con respecto al grupo 1 (G1) o grupo control. Estos resultados, ponen de manifiesto las dificultades que presentaron los grupos 2 y 3 , en las habilidades referentes a la cognición social.

$\mathrm{Al}$ analizar la variable de percepción de las expresiones emocionales (tarea visuoperceptiva/lectura de miradas) en los grupos 2 y 3 , se observaron los siguientes aspectos: ambos grupos presentaron una incapacidad para reconocer estados mentales, particularmente en la identificación de emociones, tanto básicas, como las emociones complejas que se expresen mediante la presentación visual de una mirada; de igual modo mostraron dificultades para identificar estados epistémicos o cognitivos que se expresen a través de una mirada; ambos grupos muestran incapacidad para reconocer el significado léxico complejo que hace referencia a emociones y sentimientos.

Las dificultades en la percepción de emociones que han sido halladas en los grupos conformados por niños con TDAH (G2), se han corroborado en estudios como los de Roselló-Miranda et al. (2016), Sánchez-Pérez y González-Salinas (2013), García y Hernández (2010), López-Martín, 
Albert, Fernández-Jaén, y Carretié (2010), Barkley, Murphy y Fischer (2010); Yuil y Lion (2007), en los cuales se confirman las dificultades que tiene esta población en el procesamiento de contenidos afectivos, no solo por las incapacidades cognitivas generales que manifiestan, sino por la discapacidad primaria que tienen en los procesos de identificación y comprensión de las expresiones faciales de emociones.

En el caso de los niños con TDAH/TOD (G3), las dificultades de percepción de estados emocionales, se han asociado a la forma como interactúan con el medio ambiente, según Barry, Lochman, Fite, Wells y Colder (2012) las relaciones interpersonales que establecen los llevan a usar comportamientos coactivos, agresivos y destructivos, dificultándoles relacionarse con las personas a su alrededor. En consecuencia, los niños comienzan a presentar modos problemáticos de procesar el contenido social que extraen de las otras personas, por lo cual llegan a confundir o a no identificar las emociones y pensamientos en los demás individuos.

Estas dificultades en las habilidades sociales de esta población se han relacionado con déficits en competencias de la cognición social como la asertividad, la cooperación, autocontrol, percepción y comprensión de relaciones lógicas y casuales de situaciones sociales (Díez, García - Sánchez, Robledo y Pacheco, 2009), lo que genera alteraciones en las relaciones interpersonales y manejo en el estado emocional (Jara, García-Castellar y Sánchez-Chiva, 2011).

Otras investigaciones han hecho referencia a que los niños con TDAH presentan un déficit en el proceso comunicativo no verbal, en específico en el proceso perceptivo, en este sentido, el reconocimiento de expresiones faciales de emociones se vería afectado, por lo que su correcta identificación resulta fundamental para establecer y regular relaciones interpersonales (Pardos et al., 2009).

Los resultados encontrados se vinculan con las dificultades en las competencias sociales y cognitivas encontradas en niños, como son la capacidad de flexibilidad de conducta frente a los cambios, la autorregulación, la automonitorización, la resolución de problemas, el comportamiento autónomo, la planificación de conductas dirigidas hacia un objetivo, la iniciativa, la toma de decisiones, la fluidez de pensamiento, la inhibición de respuestas inmediatas, etc. (Russo, Arteaga, Rubiales \& Bakker, 2015). 
De igual forma, la investigación de Rodrigo-Ruiz, Pérez-González y Cejudo (2017) confirma la dificultad que presentan los individuos con TDAH en las tareas que implican la detección de las expresiones emocionales, los autores corroboraron que los niños con TDAH tiene mayores dificultades, que los grupos controles, a la hora de reconocer emociones negativas, como por ejemplo la ira, miedo e incluso asco.

En otros estudios se han propuesto correlaciones negativas entre las variables de inteligencia emocional y la sintomatología asociados al mismo trastorno. Así, los niños diagnosticados con TDAH manifiestan un bajo desarrollo en varias facetas de la inteligencia emocional.

Al analizar la variable percepción del comportamiento social (test de falso paso) en los grupos 2 y 3 , se encontró que los niños manifestaron dificultades en la detección de conductas inapropiadas en el marco de una situación determinada, pudiendo causar una molestia a otra persona; interpretación de acciones, palabras, dobles sentidos, intenciones, entre otros aspectos; comprensión de los sucesos y acontecimientos de una situación específica; predicción del impacto emocional que una acción produce en otra persona.

Las dificultades antes mencionadas que manifestaron los sujetos de los grupos 2 (TDAH) y 3 (TDAH/TOD), se han relacionado con los déficits en el proceso de interpretación de información social y la atención a pistas relevantes, a la capacidad de aportar soluciones hipotéticas a situaciones sociales o problemas sociales. Igualmente, se hace referencia a la incapacidad que presentan al dar uso y aplicación de dichas pistas ambientales, lo cual lleva a dificultar la orientación de su funcionamiento social en función de los diversos contextos y condiciones (Pardos et al., 2009).

Así mismo, los estudios realizados por Jara et al. (2011), apuntan a la presencia de dificultades en el comportamiento social y por ende en las relaciones sociales en niños con estas patologías; por tanto, podrían inadecuadamente: interpretar los comportamientos sociales de otros, identificar un problema o conflicto social, así como propiciar soluciones efectivas y designar un valor a las consecuencias. Esto se vio reflejado en la ejecución de las pruebas, cuando no lograban reconocer las conductas inadecuadas de los personajes de las historias, no fueron capaces de entender, muchas veces, las razones o motivos de las acciones y no reconocían el impacto que causaba la conducta inapropiada a una tercera persona. 
Estas incapacidades encontradas en los niños con TDAH Y TDAH/ TOD podrían asociarse con el inadecuado desarrollo de las habilidades comunicativas verbales y no verbales, siendo así que, la dificultad radicaría en que ambos participantes de una interacción social, posean conocimiento previo, presunciones de la otra persona en la conversación, así como las intenciones al comunicar una información (Sperber y Wilson, como son citados en Baron-Cohen, 1990). Esto lleva a considerar que la atribución del estado mental de los niños con trastornos de conducta se afecta al tratar de comprender y respetar normas y reglas en el diálogo con las otras personas, es decir, al ser consciente de la manera como se expresa y designa un contenido social.

Por otro lado, las inhabilidades presentadas se han apuntado a dificultades en el lenguaje. En estudios actuales se han analizado las dificultades en el uso de los marcadores conversacionales y de la pragmática en los niños con TDAH (Giraldo y Castaño, 2015). Lo anterior, lleva a suponer que a los niños con estas patologías les cuesta percibir la intención comunicativa de su interlocutor y presentan una narración compleja de seguir por el interlocutor, llevando a que valoren de forma incorrecta las conductas emocionales en una conversación.

Estas dificultades en el lenguaje narrativo y pragmático se han relacionado con déficit en el sistema ejecutivo, lo que no permite una planificación en la narración en base a las necesidades del interlocutor, y de esta forma, generar una comprensión adecuada (Miranda-Casas, Ygual-Fernández \& Rosel-Remirez, 2010). Igualmente, esta investigación fundamenta que quienes padecen este trastorno presentan dificultades en la interacción social. Por tanto, puede influir en las relaciones interpersonales, haciendo que las relaciones sean caóticas, estén en decaimiento, con irrespeto de pensamientos, entre otros aspectos.

Por otro lado, al hallar déficit de percepción de emociones y comportamiento social en los niños con TDAH y TDAH/TOD en tareas de Teoría de la mente, se podría analizar que el compromiso que tienen estos niños (TDAH y TDAH/TOD) en la corteza orbitofrontal y medial, ocasiona dificultades en los procesos de mentalización, generando debilidades al realizar atribuciones mentales de los demás y la anticipación de respuestas, habilidades fundamentales para la cognición social. De acuerdo con lo descrito, el individuo con este tipo de problemáticas presenta una dificultad 
en la capacidad cognitiva para reconocer estados mentales en la otra persona (Orozco \& Zuluaga, 2015).

Con respecto a la medición entre los grupos 2 y 3, se evidenció que no existen diferencias significativas entre ellos. Estos resultados ponen de manifiesto que las alteraciones en cognición social presentada en los niños con TDAH no se ven incrementadas de manera significativa por la presencia del TOD como trastorno comórbido, pues los procesos que intervienen en la precepción adecuada de emociones y comportamiento social, tienen como base neurológica un déficit marcado a nivel prefrontal. La patología de base, por ser la misma en ambos grupos de estudio (TDAH y TDAH/ TOD), constituye una de las razones por las cuales el bajo rendimiento en tareas de cognición social, se halla presentado en igual medida en ambos grupos.

Los resultados encontrados demostraron una incapacidad notoria para detectar conductas indeseables y/o inadecuadas frente a una determinada situación social; en los niños con TOD, a nivel clínico, se evidencia en las respuestas emocionales y comportamentales como el rencor, la baja tolerancia a la frustración, molestias y resentimiento constante; esto les podría generar un mal uso del lenguaje, dificultades académicas, expresiones verbales negativas y conductas de victimización ante situaciones sociales, acusando a otros de su comportamiento (Rabadán \& Giménez, 2012).

De esta manera, el TDAH y TOD pueden ser disimiles en cuanto al tipo de respuesta que manifiestan los niños frente a una determinada situación social, no obstante, el sesgo en la precepción de emociones y comportamiento social de ambas patologías es el mismo, lo cual se refleja en el bajo desempeño obtenido en las tareas de cognición social.

\section{Conclusiones}

La capacidad adaptativa del hombre ha estado mediada por su habilidad para relacionarse y entender las emociones, deseos e intenciones de los demás a partir de las propias experiencias. La cognición social constituye uno de los procesos más complejos que le permite al individuo ponerse en el lugar de otros, predecir sus comportamientos y actuar en concordancia con los mismos. Diversas patologías que tienen como base 
una disfunción a nivel prefrontal, suponen déficit en dichos procesos, tal es el caso del TDAH y TDAH/TOD. A partir de los resultados obtenidos en la presente investigación, se concluye que:

- Los niños con TDAH/TOD muestran dificultades en la percepción adecuada de expresiones emocionales y comportamiento social en tareas de Lectura de miradas y Faux Pas (metida de pata).

- Los niños con TDAH y TDAH/TOD muestran dificultades para comprender la intencionalidad de un interlocutor, entender la pragmática del lenguaje comunicativo, así como reconocer expresiones emocionales y definirlas a través de la mirada.

- Los niños con TDAH y TDAH/TOD presentan un menor rendimiento en tareas de cognición social en relación a los niños sin estos trastornos.

- La presencia de TOD en el TDAH no afectó de manera significativa el rendimiento de los sujetos en tareas de cognición social.

- Al analizar las dos variables de la cognición social: percepción emocional y percepción del comportamiento social en los grupos de estudio 2 y 3 , los resultados de rendimiento inferior pueden deberse a que los sujetos con TDAH y TDAH/TOD presentan una menor eficacia para resolver tareas de teoría de la mente, que impliquen la capacidad de percibir señales sociales relevantes del contexto (situaciones de conflicto, engaño, doble sentido, emisión de palabras, confusiones, etc.) y de expresiones faciales (inferencia de estados emocionales y cognitivos como alegría, miedo, vergüenza, culpa, escéptico, pensativo, etc., a través de la percepción visual de una mirada), impidiendo una adecuada planificación de las acciones en relación con los propósitos que tienen.

Las investigaciones futuras habrán de considerar más variables capaces de influir en la adaptación social de los niños con TDAH, como los déficits en la regulación emocional. Concretamente, la labilidad emocional se considera un componente mediador en la relación entre el TDAH y las relaciones sociales, teniendo mayor poder de predicción en los problemas de comportamiento y de relación con iguales que los síntomas nucleares del trastorno. 
Las principales limitaciones del estudio se refieren al tamaño de la muestra clínica, en este sentido, se puede considerar como un estudio de resultados preliminares, con lo cual sería interesante ampliar y profundizar posteriormente con un mayor número de casos.

Finalmente, la presente investigación subraya la necesidad de ampliar y profundizar en una nueva línea de investigación sobre el estudio de la cognición social en los trastornos de conducta, teniendo en cuenta muestras más grandes de la población, que permita la generalización del conocimiento y la implementación de nuevos paradigmas de estudio. Igualmente, como principal aplicación, además de las ampliaciones posibles del estudio, está la consideración de los resultados en la elaboración de estrategias de intervención en competencias sociales en niños con estas patologías.

\section{Referencias}

Ashford, J. B., \& LeCroy, C. (2009). Human behavior in the social environment: A multidimensional perspective. Nelson Education. USA.

Barkley, R. A., Murphy, K. R., \& Fischer, M. (2010). ADHD in adults: What the science says. Guilford Press. New York: London.

Baron-Cohen, S. (1990). Autism: A Specific Cognitive Disorder of \& lsquo; Mind-Blindness'. International Review of Psychiatry, 2(1), 81-90.

Baron-Cohen, S., \& Wheelwright, S. (2004). The empathy quotient: an investigation of adults with Asperger syndrome or high functioning autism, and normal sex differences. Journal of Autism and Developmental Disorders, 34(2), 163-75. Recuperado de http://www.ncbi. nlm.nih.gov/pubmed/15162935.

Baron-Cohen, S., Wheelwright, S. \& Hill, J. (2001). The 'Reading the mind in the eyes' test revised version: A study with normal adults, and adults with Asperger Syndrome or High-Functioning autism. Journal of Child Psychology and Psychiatry, 42: 241-252.

Barry, T. D., Lochman, J. E., Fite, P. J., Wells, K. C., \& Colder, C. R. (2012). The influence of neighborhood characteristics and parenting practices on academic problems and aggression outcomes among moderately to highly aggressive children. Journal of Community Psychology, 40(3), 372-379. 
Butman, J., Abel, C., y Allegri, R. (2003). Bases cognitivas de la conducta social humana. Documento del II Congreso Internacional de Neuropsicología en Internet. Recuperado de http://www.psiquiatria.com/ articulos/psiq_general_y_otras_areas/psiqsocial/12044/.

Castaño, F. G. (1999). El deterioro de las habilidades sociales en función del TDAH. Ábaco, 21-28.

Clark, C.; Prior, M. \& Kinsella, G. (2002). The relationship between executive function abilities, adaptive behaviour, and academicachievement in childrenwith externalising behaviour problems. Journal of Child Psychology and Psychiatry 43(6), pp.785-796.

Díez, M. C., García-Sánchez, J. N., Robledo, P., \& Pacheco, D. I. (2009). Habilidades sociales y composición escrita en alumnos con dificultades de aprendizaje y/o bajo rendimiento. Boletín de Psicología, 95, 73-86.

Eme, R. F. (2007). Sex differences in child-onset, lifecourse-persistent conduct disorder. A review of biological infl uences. Clinical Psychology Review, 27, 607-627.

Extremera, N., \& Fernández, P. (2003). La inteligencia emocional en el contexto educativo: hallazgos científicos de sus efectos en el aula. Revista de educación, 332(2003), 97-116.

Fernández- A, López S, Albert J, Martín D, Fernández, A. y Calleja, (2017). TDAH: perspectiva desde el neurodesarrollo. Rev Neurol 2017; 64 (Supl 1): S101-4.

Fonseca-Parra, L., \& Rey-Anacona, C. (2013). Características Neuropsicológicas De Niños Con Trastorno Negativista Desafiante. Revista De Psicopatología Y Psicología Clínica 18(3), 233-244.

García, A. M., \& Hernández, S. (2010). Alteraciones emocionales en el trastorno por déficit de atención con Hiperactividad. Revista de psiquiatría infanto-juvenil, 27(1), 17-24.

García, F. (1999). El deterioro de las habilidades sociales en función del TDAH. Abaco: Revista de cultura y ciencias sociales. 21-22 Pp. 2128.

Giraldo, Y. G., \& Castaño, L. C. (2015). Trastorno por déficit de atención/ hiperactividad (TDAH) y dificultades en lenguaje pragmático. Revista de Psicología Universidad de Antioquia, 6(1), 41-56. 
Hernández, R., Fernández, C., \& Baptista, M. (2014). Metodología de la Investigación. Sexta ed., México: McGraw Hill.

Hurtado de Barrera, J. (2012). El proyecto de investigación. Una comprensión holística.

Jara, P., García Castellar, R., \& Sánchez Chiva, D. (2011). Competencias sociales de niños con trastorno por déficit de atención e hiperactividad "TDAH": valoración desde el entorno familiar y escolar. Quaderns Digitals, 69.

López-Martín, S., Albert, J., Fernández-Jaén, A., \& Carretié, L. (2010). Neurociencia afectiva del TDAH: Datos existentes y direcciones futuras. Escritos de Psicología (Internet), 3(2), 17-29. Recuperado de http://scielo.isciii.es/scielo.php?script=sci_arttext\&pid=S1989-38092010000100003\&lng=es\&tlng=es.

Mellado, M., Martínez, L., \& Tello, F. (2013). Procesos atencionales implicados en el Trastorno por Déficit Atencional con Hiperactividad (TDAH). Convergencia educativa, (2), 9-19.

Miranda-Casas, A., Ygual-Fernández, A., \& Rosel-Remírez, J. (2004). Complejidad gramatical y mecanismos de cohesión en la pragmática comunicativa de los niños con trastorno por déficit de atención con hiperactividad. Rev Neurol, 38(Suppl 1), S111-6.

Montoya., P \& Arango, O., (2015). ¿Cómo es la relación entre cognición social y trastorno disocial de la conducta? Editorial: Fundación Universitaria Luis Amigó. Facultad de Psicología y Ciencias Sociales Grupo de Investigación Neurociencias Básicas y Aplicadas. Recursos Electrónico. Medellín, Colombia.

Orjales, I. (2007). El tratamiento cognitivo en niños con trastorno por déficit de atención con hiperactividad (TDAH): revisión y nuevas aportaciones. Anuario de Psicología Clínica y de la Salud, 3, 19-30.

Orozco, N., \& Zuluaga, J. (2015). Teoría de la mente en niños y niñas con trastorno por Déficit de Atención con Hiperactividad "TDAH". Tesis Psicológica, 10(2), 134-148.

Pardos, A., Fernández-Jaén, A., \& Fernández-Mayoralas, D. M. (2009). Habilidades sociales en el trastorno por déficit de atención/hiperactividad. Rev neurol, 48(Supl 2), S107-11. 
Penn, D.L., Corrigan, P.W., Bentall, R.P., Racenstein, J.M. y Newman, L. (1997). Social cognition in schizophrenia. Psychological Bulletin, $121,114-132$.

Perner, J. \& Lang, B. (1999). Development of theory of mind and executive control.

Pineda, W., \& Puentes, P. (2013). Estudio ex-post facto de la teoría de la mente en niños escolarizados diagnosticados con trastorno por déficit de atención-hiperactividad. Tesis Psicológica, 8 (2), 144-161.

Rabadán Rubio, J., \& Giménez Gualdo, A. (2012). Detección e intervención en el aula de los trastornos de conducta. Educación XX1, 15(2), 185-212.

Ramírez, M. (2015). Tratamiento cognitivo-conductual de conductas disruptivas en un niño con TDAH y trastorno negativista desafiante. Revista de Psicología Clínica con niños y adolescentes, 2(1), 4554.

Rapport LJ, Friedman SR, Tzelepis A., \& Van Voorhis A. (2002). Experienced emotion and affect recognition in adult attention-deficit hyperactivity disorder. Neuropsychology 2002; 16: 102-10.

Restrepo, J., Arana C., Alvis, A., Gómez A., y Hoyos, E. (2015). Relación entre Cognición Social y Funciones Ejecutivas en Niños con Trastorno Negativista Desafiante. Revista Iberoamericana de Diagnóstico y Evaluación - e Avaliação Psicológica. RIDEP . 42(2) · 49-58.

Rodrigo-Ruiz D, Pérez-González JC, Cejudo J. (2017). Dificultades de reconocimiento emocional facial como déficit primario en niños con trastorno por déficit de atención/ hiperactividad: revisión sistemática. Rev Neurol; 65: 145-52.

Rodríguez, J., Acosta, M. y Rodríguez. (2011). Teoría de la mente, reconocimiento facial y procesamiento emocional en la esquizofrenia. Revista de psiquiatría y salud mental, 4(1), 28-37.

Roselló-Miranda, B., Berenguer-Forner, C., Baixauli-Fortea, I. \& Miranda-Casas, A. (2016). Un modelo integrador de la adaptación social de niños con TDAH. Memorias de XVIII Congreso sobre Trastornos del Neurodesarrollo. Recuperado de http://horarioscentros.uned. es/archivos_publicos/qdocente_planes/1037973/rosellomirandaetal2016modelointegradordelaadaptacionsocialdeninoscont.pdf. 
Rubia, K. (2011). «Cool» inferior frontostriatal dysfunction in Attention-Defi cit/Hyperactivity disorder versus «hot» ventromedial orbitofrontal-limbic dysfunction in Conduct Disorder: A review. Biological Psychiatry, 69, 69e-87e.

Ruiz-Ruiz, J. C., García-Ferrer, S., \& Fuentes-Durá, I. (2006). La relevancia de la cognición social en la esquizofrenia. Apuntes de psicología, 24(1-3), 137-155.

Russo, D., \& Arteaga, F., \& Rubiales, J., \& Bakker, L. (2015). Competencia social y status sociométrico escolar en niños y niñas con TDAH. Revista Latinoamericana de Ciencias Sociales, Niñez y Juventud, 13(2), 1081-1091.

Sánchez-Pérez, N., \& González-Salinas, C. (2013). Ajuste Escolar del Alumnado con TDAH: Factores de Riesgo Cognitivos, Emocionales y Temperamentales. Electronic Journal of Research in Educational Psychology, 11(2), 527-550.

Stone, V. E., Baron-Cohen, S. \& Knight, R. T. (1998). Frontal Lobe Contributions to Theory of.

Yuill, N. \& Lyon, J. (2007). Selective difficulty in recognising facial expressions of emotion in boys with ADHD. General performance impairments or specific problems in social cognition? European Child Adolescent Psychiatry, 16, 398- 404. 